\title{
Safety, mobility and comfort assessment methodologies of intelligent transport systems for vulnerable road users
}

\author{
Kerry Malone $^{1}$ (D) $\cdot$ Anne Silla $^{2} \cdot$ Charlotta Johanssen $^{3} \cdot$ Daniel Bell $^{4}$
}

Received: 21 May 2016/Accepted: 17 March 2017 /Published online: 17 April 2017

(C) The Author(s) 2017. This article is an open access publication

\begin{abstract}
Introduction This paper describes the modification and development of methodologies to assess the impacts of Intelligent Transport Systems (ITS) applications for Vulnerable Road users (VRUs) in the domains of safety, mobility and comfort. This effort was carried out in the context of the VRUITS project whose aim was to produce results at the EU-28 level. Methods An existing safety methodology was modified to take into account specific VRU aspects. The mobility and comfort assessments methodologies were developed in the project. Results The safety, mobility and comfort methodologies were applied to ten ITS applications for VRUs. The first innovation determined how the nine safety mechanisms for ex-ante analysis of ITS applications, including direct and indirect effects, can incorporate the important characteristics of the VRU groups (pedestrians, cyclists and Power-Two-Wheeler riders) in the analysis. The second innovation developed a conceptual model for mobility and comfort. Thirdly, the estimation of quantitative effects, using literature, empirical findings and expert judgement, was developed.

Conclusions The new safety, mobility and comfort assessment methodologies were applied to calculate the respective effects for VRUs using ITS. These results are ex-ante findings,
\end{abstract}

This article is part of Topical collection on The Influence of Intelligent Transport Systems on Vulnerable Road User Accidents

Kerry Malone

kerry.malone@tno.nl

1 TNO, The Hague, The Netherlands

2 VTT, Espoo, Finland

3 Lulea University of Technology, Lulea, Sweden

4 FACTUM, Wien, Austria as very few to no empirical results for ITS applications for VRUs are available. In order to improve the accuracy of the estimates, there is a need for better standardized data and at the European level. Finally, validation of the methods could be done in the future field operational tests focusing on measuring user behavior.

Keywords Vulnerable road users · (cooperative) intelligent transport systems $\cdot$ Impact assessment $\cdot$ Methodology Safety $\cdot$ Mobility $\cdot$ Comfort

\section{Introduction}

In recent years both technological developments and research activities in the fields of Intelligent Transport Systems (ITS) have primarily focussed on motorised transport to improve safety and ecological (environmental) impacts by advancing equipment of vehicles and infrastructure. The deployment of ITS applications, such as Electronic Stability Control (ESC), has assisted in the decrease of road traffic fatalities, particularly amongst passenger car occupants $[9,20]$. However, vulnerable road users such as pedestrians, cyclists, motorcyclists and moped riders have not enjoyed a comparable decrease in fatalities. Figure 1 shows the number of fatal accidents in the EU in the 10-year period 2002-2012. Passenger car fatalities have declined steeply over the period whereas the decline for the VRU groups is again much less pronounced, with bicycle fatalities showing a negligible decline. While some projects have considered VRUs from a safety viewpoint, they often aimed at avoiding or mitigating accidents with VRUs by equipping the vehicle and infrastructure. In the vehicle - infrastructure human approach of ITS research, VRUs and their needs are not an active part of the "human" element in the ITS approach. 
Fig. 1 Trends in numbers of accidents (Fatalities), 2002 to 2012 [8]

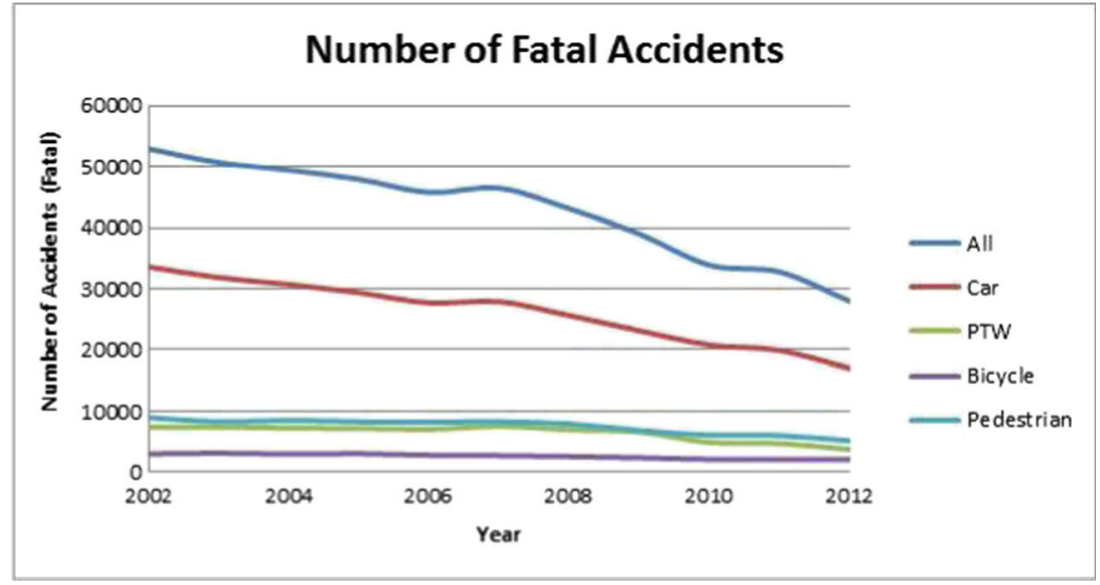

What is needed? The VRU must become an active, integrated element in the ITS, addressing safety, mobility and travel comfort needs of VRUs. The EU-funded VRUITS project, which ran from April, 2013, through March, 2016, aimed at actively integrating the "human" element in the ITS approach by focussing on the needs of all relevant stakeholder groups in the development and adaptation process of innovative ITS solutions aimed at improving the traffic safety, mobility and comfort of vulnerable road users. The VRUITS project, which was sponsored by the European Commission DG MOVE, placed the vulnerable road user in the centre, assessed the impact of current and upcoming ITS applications on the safety, mobility and comfort of vulnerable road users, identified how the usability and efficiency of ITS applications could be improved, and recommended which actions have to be taken at a policy level to improve ITS safety and mobility. By applying a multi-disciplinary approach, the VRUITS project aimed at developing tools to evaluate, field-test and subsequently improve ITS for vulnerable road users. Consistent with white papers and goals of the European Commission defined for 2020 and 2030, the quantitative estimates were produced for these years (but not presented in this paper). See [1] for results.

Part of the first objective of the VRUITS project, and the objective directly related to this paper, is to assess societal safety, mobility, comfort and cost-benefit - impacts on vulnerable road users of selected ITS applications. In this paper, "impact assessment" will be used to refer to the set of safety, mobility, comfort and cost-benefit assessments. The impact assessment for vulnerable road users of ITS is new.

The objective of this research paper is to describe the methodologies and data used in the impact assessment for vulnerable road users. Because the methodologies are new and the evidence for impacts of the investigated ITS is scarce or nonexistent, the methodologies must be innovative in their approach and in finding the data to quantify the safety, mobility and comfort impacts. This paper focusses on providing a detailed description of these aspects. Firstly, the paper introduces the vulnerable road user categorisation used in VRUITS. The safety, mobility and comfort methodologies are then presented in separate sections. For each of these impact areas, an introduction to the state-of-the-art methodologies for that area is presented, followed by a justification of the method chosen for assessment. Each section then describes how the method is modified to take into account vulnerable road users, as well as the data needs of the method. The paper concludes with a reflection on the methods and data.

\section{Vulnerable road user categorisation}

Which road users are vulnerable? Although all road users are at risk of being injured or killed in a road traffic accident, certain groups of road users are at a higher risk and the fatality rate of accidents varies between the different road user groups. In particular, the "vulnerable" road users such as pedestrians and cyclists (7-9 times higher than car passengers, measured per $\mathrm{km} \mathrm{[5]),} \mathrm{and} \mathrm{also} \mathrm{motorcyclists} \mathrm{(approximately} 20$ times higher than car passengers, measured per $\mathrm{km} \mathrm{[5]),} \mathrm{are} \mathrm{at} \mathrm{great-}$ er risk than vehicle occupants.

There are different definitions of vulnerable road users in use. [15] summarizes the definitions of vulnerable road users. The definitions consider road users vulnerable if they lack external protection. VRUITS uses roughly the same three criteria to distinguish vulnerable road users as [18] as a basis for classifying the road user groups. They are:

- The amount of external protection

- Task competency, i.e., the extent to which people are able to function in risky situations

- Resilience (fragility), i.e., the extent to which people can absorb outside forces

[18] provides a detailed description of the three criteria above. 
The absence of a protective cage leads to a mode-related identification of vulnerable road users: pedestrians, cyclists, moped riders and motorcyclists. Fragility leads to the further identification of children and elderly as sub-groups within some of the mode-related classification. Finally, task competency affects the way and (in)ability of some subgroups to function in traffic, which is a risky situation. The age of a road traffic participant determines to a large part the task competency of that individual. A review of children's characteristics as road users revealed that there are appropriate age groups with specific needs concerning the right to survive (safety), to move (mobility and comfort) and to take part in the society. Safety, mobility and comfort are all important concepts which often go or at least should go "hand in hand". For the elderly (older than 64 years), these physical and cognitive resources decline with age, but there can be significant individual differences in abilities and in the behaviour of elderly. The variation in individual health and physical abilities can be great for elderly persons of the same age [18].

Using the categorisation summarised above, VRUITS used the age structures of vulnerable road users shown in Table 1, when possible.

Combining the presence of a protective cage and age categorization of vulnerable road users, the VRUITS project created relevant age subcategories within each mode (pedestrians, cyclists, moped and motorcyclists).

\section{Safety impact assessment}

\subsection{Summary of state-of-the-art safety impact assessment methodologies for ITS}

Summarising the extensive literature review of [14], the following methods can be distinguished for an ex-ante and expost evaluation of active safety systems:

- Safety mechanisms: The safety mechanism approach is summarised in [14] as follows: the framework of a safety assessment of ITS should (1) cover all three dimensions of road safety - exposure, crash risk and consequence, (2) cover the effects due to behavioural adaptation in addition to the engineering effect (effect on target accident contributory factors) and (3) be compatible with the other aspects

Table 1 Categorisation of age structures of vulnerable road used in VRUITS

\begin{tabular}{ll}
\hline Description & Age in years \\
\hline Younger children & $7-12$ years \\
Older children; teens & $13-17$ years \\
Adult & $18-64$ years \\
Elderly & $65+$ years old \\
\hline
\end{tabular}

of state of the art road safety theories. This entails an estimation of the target population of the ITS and an expert evaluation of its effectiveness in preventing or mitigating accidents. A framework for assessing the road safety impacts that fulfils these requirements is the nine-point list of ITS safety mechanisms.

- Expert Questionnaires

- Accident reconstruction: this is based on case study-approach, where accident scenarios are simulated with and without the ITS present, and the outcomes are compared.

- Black box statistical analysis: a method based on artificial neural networks that assesses safety-based on information about the relevance and influence of the ITS on accident characteristics.

- Ex-post evaluation: this is based on accident data with and without the ITS.

- Field Operational Test data analysis: This approach uses Field Operational Test data to assess safety. The analysis uses data on near accidents or risky events and translates that data into an estimate on safety.

- Effectiveness methodology using a tree approach: This approach is based on mapping an accident database to a tree to classify the conditions of the injuries. The mapped accidents are multiplied by percentage of road users that didn't not die/injured to estimate the effect of an ITS measure.

The methodologies reviewed above have hardly been applied to vulnerable road users. Methods based on ex-post analysis of accident statistics are not relevant for the case of ITS for vulnerable road user, because little or no statistical data on such applications are available. The lack of statistical data also precludes the use of models with "predictive" ability, using data about the past. This is due to the ITS being a "trend break" rather than a measure for which the impacts can be predicted. Thus, ex-ante assessment methods seem more appropriate. A comprehensive approach that covers all possible safety effects is the safety mechanisms method, and therefore this was used in the VRUITS project.

\subsection{Overview of method development}

The starting point for the development of the safety impact assessment method which was used in VRUITS project was the safety impact assessment framework presented by [15]. The framework of [15] is based on the theoretical background presented by [19] according to which the traffic safety consists of three dimensions, which are (1) exposure, (2) risk of a collision to take place during a trip and (3) consequences (= risk of a collision to result in injuries or death) ([19], as illustrated in Fig. 2). The volume of the rectangular box is the expected number of injured or fatalities. Thus the number of 


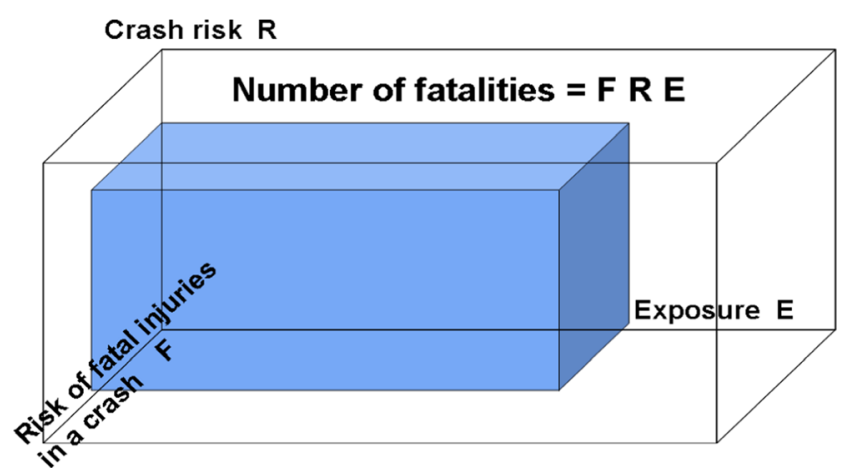

Fig. 2 The dimensions of road safety [19]

injuries or fatalities in road accidents depends on the three dimensions - exposure, accident rate and injury severity.

The framework of [15] emphasises the systematic nature of transport: when one element of the system is affected, the consequences may appear in several elements and levels of the system. Therefore, the implemented measures influence safety by affecting one or several of the factors contributing to any of these three dimensions of safety. The use of this approach ensures that the safety impact assessment method will cover all dimensions of road safety, also exposure or the amount of travelling, which is frequently overlooked in the safety assessment studies [15].

In addition to the three dimensions of road safety (as indicated in Fig. 2) the framework for the safety impact assessment of ITS should also cover the effects due to behavioural adaptation in addition to engineering effects, and be compatible with other aspects of state-of-the-art road safety theories [15]. In order to be sure that all possible impacts (both positive and negative impacts on road safety; direct, indirect and unintended effects of systems) will be covered, the analyses proposed by [15] utilises a set of nine mechanisms via which ITS can effect road user behaviour and thereby road safety. These nine mechanisms cover the three aspects of road safety in a systematic manner and are based on a ten-point list compiled by [3]. For the purposes of the VRUITS project, these nine mechanisms were updated to cover vulnerable road users, i.e. pedestrians, cyclists, moped riders and motorcyclists. The updated mechanisms, which are now more focused on the changes in behaviour of vulnerable road users and the situations they face in traffic [29], are presented below:

\section{Mechanism 1: Direct modification of the task of road} users by giving information, advice, and assistance or taking over part of the task. This may influence their attention, mental load, and decision about action (for example, driver/rider/cyclist/pedestrian choice of speed). The criterion for this mechanism is that the effects are direct consequences of the use of the system; they are direct reactions to the system outputs and appear in few milliseconds or seconds. This mechanism covers both intended (e.g. decrease of speed to avoid a collision) and unintended (e.g. driver/rider/cyclist/pedestrian distraction) impacts. An example of such mechanism is when motorcyclists receive a warning (and a request to reduce their speed if needed) when a sharp curve is ahead.

2. Mechanism 2: Direct influence by roadside systems, mainly by giving information and advice. Without the possibility to control the road users' action or the vehicle directly, the impact of this mechanism is more limited than that of the in-vehicle systems provided as examples in mechanism 1. In other aspects the impacts are similar to the ones described in mechanism 1. An example of such a system is intelligent traffic lights which prioritise pedestrians over other road users, and therefore reduce crossing at a red light. When the waiting time is shorter, the road users are expected to respect the red light better.

3. Mechanism 3: Indirect modification of user behaviour in many, largely unknown, ways. The driver/rider/cyclist or the pedestrian will always adapt to the changing situation. This is called behavioural adaptation, and will often not appear immediately after introduction of a new system but may show up later. It is very hard to predict. The indirect modification is more long-term than the very direct, shortterm reactions to the system in mechanisms 1 and 2 . Examples include overreliance on warnings provided by the system, resulting in road users observing their surroundings less carefully.

4. Mechanism 4: Indirect modification of non-equipped road user behaviour. This type of behavioural adaptation is even harder to study because it is often secondary. Nonequipped road users may for example change their behaviour by imitating the behaviour of equipped road users (for example riding closer or faster than they should, without the equipment).

5. Mechanism 5: Modification of interaction between equipped road users and non-equipped road users. ITS will change the communication between equipped road users. This change of communication may also influence the traditional communication with non-equipped road users. To a large extent this problem may appear in the interaction between drivers and unprotected road users.

6. Mechanism 6: Modification of road user exposure due to information, recommendations, restrictions or increased comfort in car driving, Power-Two-Wheeler riding, cycling or walking. This mechanism covers only changes in the amount of travelling, i.e. whether the road user decides to make more or fewer, or longer or shorter, trips due to the system.

7. Mechanism 7: Modification of modal choice by, for example, demand restraints (area access restriction, road pricing, area parking strategies), supply control by modal interchange and other public transport management measures, and travel information systems. Different travel 
modes have different accident risks, therefore any measure which influences modal choice, also has an impact on road safety. Traffic information can lead to travellers choosing a safer mode, i.e., modify modal choice.

8. Mechanism 8: Modification of route choice by route diversions, route guidance systems, dynamic route information systems, and hazard warning systems monitoring incidents. Different parts of the road network, i.e., different categories of roads, have different accident risks. Therefore, any measure which influences route choice by directing or diverting traffic to roads of a different category, also has an impact on road safety. Note that route changes also affect exposure, and the exposure changes due to the route changes can be taken into account either under this mechanism or mechanism 6 (exposure).

9. Mechanism 9: Modification of accident consequences by intelligent injury severity-reducing systems activated when the vehicle crashes into another vehicle or into a pedestrian, by quick and accurate crash reporting and call for rescue, and by reduced rescue time.

As indicated by [15] many of these mechanisms are closely linked to one another, and could be combined. Examples of these are mechanisms of direct driving behaviour modification (1-2), indirect driving behaviour modification (3-5), and travel pattern modification (6-8). The mechanisms have not, however, been combined since the purpose of the framework is to illustrate the types of different possible effects (both positive and negative) of IT systems on safety.

\subsection{Accident data}

In the VRUITS project the accident data was handled similarly as in the INTERSAFE2 project [30] and in the eIMPACT project [30]. The accident data in the eIMPACT project covered the EU-25 countries from 2005 [31] and the INTERSAFE2 project updated the accident data to include the new member countries Romania and Bulgaria and extended the database to the EU-27. In the VRUITS project the accident data was further extended to cover the EU-28. The CARE database [2] was chosen for the analysis due to its European coverage. The total number of fatalities and injuries used in the calculations are presented in Table 2. The figures for 2020 and 2030 were calculated based on accident trends including separate estimates for accidents related to pedestrians, cyclists, moped riders, motorcyclists and cars [6].

The total number of fatalities used in the impact assessment calculations for the EU-28 was taken from the Statistical pocketbook [4]. The statistical pocketbook does not include any information on the number of injuries (only on the number of injury accidents) and thus the total number of injuries was taken from CARE database. Based on our analysis, the annual
Table 2 Total number of fatalities and injuries used in the calculations

\begin{tabular}{llll}
\hline & 2012 & 2020 & 2030 \\
\hline Fatalities & 28,126 & 16,428 & 8572 \\
Injuries & $1,429,888$ & $1,085,888$ & 762,262 \\
\hline
\end{tabular}

Calculated based on EC 2014 [4] and CARE database [1]

number of fatalities reported in CARE in $2012(25,738)$ matched well with the number reported in the Statistical pocketbook $(25,776)$, when taking into consideration only the countries for which 2012 data were available. Therefore we made the assumption that the number of injuries reported in CARE matched well with the numbers in the statistical pocketbook and no further correction was necessary.

More detailed information on fatalities and injuries for the EU-28 were gathered from the statistics of CARE database for the year 2012. No accident data for 2012 were available for Belgium, Bulgaria, Estonia, Lithuania, Malta, Slovakia and Sweden and thus the latest available data in CARE database was used for those countries instead (2011 for Belgium, 2010 for Malta, Slovakia and Sweden and 2009 for Bulgaria and Estonia). The CARE database included no information on road accidents for Lithuania. Thus the total numbers of fatalities and injuries in 2012 were taken from the Lithuanian national statistics. The resulting figure for fatalities in the EU-28 was 28,126 .

The data from the CARE database were used to classify the fatalities and injuries according to the following background variables: collision type, road type, weather conditions, lighting conditions, location of the accident and age. Background variables, sometimes called "situational variables", are necessary because they provide insight into whether an ITS is appropriate for certain circumstances. If not, the effectiveness of the ITS in that situation is negligible or nil. Compared to the previous projects (e.g., eIMPACT and INTERSAFE2), it was not possible to determine if the vulnerable road user had been in an accident with a heavy or light vehicle (except for pedestrian accidents). This is because CARE does not allow for the collision partner to be identified; the data are classified using one vehicle type e.g., it is possible to tell that a cyclist had an accident with multiple vehicles but it is not possible to distinguish the characteristics of the other vehicles. The distribution of fatalities and injuries according to the different background variables was exploited when calculating the safety effects of different ITS (see Section 5).

There is variability in the quality of the accident data entered into European-wide accident databases by country with some being highly detailed and accurate whereas others have many cases of 'unknowns'. To generate background variable data, the following approach was used, similar to eIMPACT. This approach groups countries with similar safety characteristics together. The accident data for the EU-25 in eIMPACT 
project [30] was divided into three clusters. The clusters were formed based on the prevalent safety situation in each country and therefore the countries with similar road safety situations were included in the same cluster. For the countries where no detailed information was available on the background variables, or when the values were not considered reliable, the average values from the cluster to which the country belonged were used. In VRUITS, these clusters were updated by using the latest road safety and vulnerable road user safety-related statistics.

Tables 3 and 4 present the distribution of vulnerable road user fatalities and injuries by collision type (Table 3 ) and the distribution of vulnerable road user fatalities and injuries by other variables (Table 4). The total number of fatalities and the total numbers of fatalities of pedestrians, cyclists, moped riders and motorcyclists were taken from the statistical pocketbook from which the share of single vehicle accidents were separated based on the information reporting in CARE database. For this work, only the information from countries that had provided the specific information was taken into consideration. Regarding injuries, the classification of injuries into different collision types was based exclusively on the data reported to the CARE database for the countries that had provided the specific information. The adverse weather conditions presented in Table 4 included fog, mist, rain, snow, sleet and hail. Twilight was included in the lighting condition Daylight. Compared to previous projects, age group was included in the analysis as an additional variable.

\subsection{Exposure effects}

In the VRUITS project, the effects of exposure (as part of Mechanism 6) focused on changes in exposure as measured in kilometres of travel for different vulnerable road user groups. The estimated effects on vulnerable road user exposure were transferred to safety effects of exposure (the same values were used for fatalities and injuries) based on the values found in earlier studies. These studies found a "safety in numbers" effect, where the accident risk per kilometre for vulnerable road user modes decreases as vulnerable road user travel increases - a likely explanation is that other road users learn to expect vulnerable road users on the road when they become less rare. This means that the number of fatalities and injuries increases slower than the number of vulnerable road user kilometres. The studies modelled this with an exponential model: if the exposure increased by a factor of $\mathrm{x}$, then the number of fatalities and injuries increased by a factor $\mathrm{x}^{\mathrm{y}}$, for some exponent $y$ less than 1 . The exponent y was 0.38 for the pedestrians (based on [12]), 0.4 for cyclists and moped riders (based on [11]) and 0.7 for motorcyclists (based on [17] and previous impact assessment studies). The safety effects of modal change (Mechanism 7) were calculated by using the same formulas as for the change (increase or decrease) in the exposure of vulnerable road users (Mechanism 6). The mechanism-based approach in our safety assessment allowed us to conclude that no double counting of the effects occurred. The effects of the modal shift were only calculated for vulnerable road users; the effects of the modal change of cars, trucks and public transport were ignored. A small change in the modal share of vulnerable road users corresponds to a significantly smaller change in the modal change of cars, trucks and/ or public transport, thus the safety effect of the modal shift to or from cars, trucks and public transport would be negligible. Moreover, the risk per $\mathrm{km}$ for cars, trucks and public transport is much smaller than the risk per $\mathrm{km}$ for vulnerable road user [5]. Thus, calculating the effect of modal change for cars, trucks and/or public transport results in a smaller change in travelled $\mathrm{km}$ that would be multiplied by a smaller risk factor per $\mathrm{km}$.

\subsection{Summary}

The method to assess the safety impacts of ITS on vulnerable road users is based on the method introduced by [15], which was developed for the assessment of safety impacts of ITS for cars. This method is aimed at ITS - as was VRUITS - and is comprehensive in its approach, covering all three dimensions of road safety - exposure, crash risk and consequence, the effects due to behavioural adaptation in addition to the engineering effect (effect on target accident contributory factors) and is compatible with the other aspects of state-of-the-art road safety theories. Some parts of the method were enhanced and adjusted to take also into consideration the vulnerable road users. The main modifications of the method for the purposes of the VRUITS project were related to: i) nine mechanisms which were updated to cover vulnerable road users i.e. pedestrians, cyclists, moped riders and motorcyclists, ii) the safety impact assessment tool which was updated to include more detailed information on accidents involving vulnerable road users, iii) accident types and circumstances such as age, road layout and lighting which were considered in more detail when relevant for vulnerable road user and when feasible, iv) the calculation of safety effects of exposure changes and v) the expert assessment which was used to enhance the value of estimates for the nine mechanisms (see section 5).

\section{Mobility and comfort impact assessments}

\subsection{Summary of state-of-the-art mobility and comfort impact assessment methodologies for ITS}

While mobility levels, levels of service and general mobility behaviour have been very active research fields, resulting in an increasing number of data sets available on both the national and international levels, the concept of comfort for 
Table 3 Distribution of fatalities and injuries by collision type

\begin{tabular}{llll}
\hline Variable & Classification & $\begin{array}{l}\text { Percentage of } \\
\text { fatalities }\end{array}$ & $\begin{array}{l}\text { Percentage } \\
\text { of injuries }\end{array}$ \\
\hline Collision type & Pedestrian accidents & $21 \%$ & $13 \%$ \\
& Single vehicle cycle accidents & $1 \%$ & $2 \%$ \\
& Multiple vehicle accidents involving cycles & $7 \%$ & $10 \%$ \\
& Single vehicle moped accidents & $1 \%$ & $1 \%$ \\
& Multiple vehicle accidents involving mopeds & $3 \%$ & $5 \%$ \\
& Single vehicle motorbike accidents & $4 \%$ & $2 \%$ \\
& Multiple vehicle accidents involving motorcycles & $10 \%$ & $8 \%$ \\
& Single accidents involving cars & $20 \%$ & $13 \%$ \\
& Other accidents with two vehicles & $34 \%$ & $45 \%$ \\
& Total & $100 \%$ & $100 \%$ \\
\hline
\end{tabular}

Calculated based on EC 2014 [4] and CARE database [1] pedestrians, cyclists and Power-Two-Wheelers is often not dealt with explicitly and in detail. Nevertheless, there are a number of studies from the fields of spatial planning, architecture and civil engineering that specifically address this topic. No clear and definitive mobility and comfort assessment methodologies for vulnerable road users are available.

There are a number of different definitions of mobility for different road users. VRUITS used the definition of mobility based on the work of [24], that links well to vulnerable road users and different functionalities of ITS: "vulnerable road user mobility is any form of outside (of the house) movement based on the identified soft transport modes: walking, cycling or motorcycling. These forms of movement are defined by trips from a starting point to a destination (where the destination can also be a public transport stop or station) in order to conduct an out-of-house activity."

Based on the literature search and review of current studies in the mobility and comfort research field, there is a severe lack of both theoretical and empirical discussion of the comfort topic. Below, the aspects that need to be taken into

Table 4 Distribution of vulnerable road user fatalities and injuries by variable including the classification of each variable

\begin{tabular}{|c|c|c|c|c|c|c|c|c|c|}
\hline \multirow[t]{2}{*}{ Variable } & \multirow[t]{2}{*}{ Classification } & \multicolumn{2}{|l|}{ Pedestrians } & \multicolumn{2}{|c|}{ Cyclists (multiple vehicle) } & \multicolumn{2}{|c|}{$\begin{array}{l}\text { Moped riders } \\
\text { (multiple vehicle) }\end{array}$} & \multicolumn{2}{|c|}{$\begin{array}{l}\text { Motorcyclists } \\
\text { (multiple vehicle) }\end{array}$} \\
\hline & & Fatalities $(\%)$ & Injuries (\%) & Fatalities $(\%)$ & Injuries $(\%)$ & Fatalities $(\%)$ & Injuries (\%) & Fatalities $(\%)$ & Injuries $(\%)$ \\
\hline \multirow[t]{4}{*}{ Road type } & Motorway & $4 \%$ & $0 \%$ & $0 \%$ & $0 \%$ & $0 \%$ & $0 \%$ & $4 \%$ & $3 \%$ \\
\hline & Rural & $28 \%$ & $9 \%$ & $48 \%$ & $15 \%$ & $48 \%$ & $16 \%$ & $56 \%$ & $24 \%$ \\
\hline & Urban & $68 \%$ & $91 \%$ & $52 \%$ & $85 \%$ & $52 \%$ & $84 \%$ & $40 \%$ & $73 \%$ \\
\hline & Total & $100 \%$ & $100 \%$ & $100 \%$ & $100 \%$ & $100 \%$ & $100 \%$ & $100 \%$ & $100 \%$ \\
\hline \multirow[t]{3}{*}{ Weather conditions } & Normal & $88 \%$ & $77 \%$ & $91 \%$ & $90 \%$ & $93 \%$ & $90 \%$ & $94 \%$ & $9 \%$ \\
\hline & Adverse & $12 \%$ & $23 \%$ & $9 \%$ & $10 \%$ & $7 \%$ & $10 \%$ & $6 \%$ & $91 \%$ \\
\hline & Total & $100 \%$ & $100 \%$ & $100 \%$ & $100 \%$ & $100 \%$ & $100 \%$ & $100 \%$ & $100 \%$ \\
\hline \multirow[t]{3}{*}{ Lighting conditions } & Daylight & $49 \%$ & $65 \%$ & $78 \%$ & $86 \%$ & $69 \%$ & $79 \%$ & $82 \%$ & $82 \%$ \\
\hline & Night & $51 \%$ & $35 \%$ & $22 \%$ & $14 \%$ & $31 \%$ & $21 \%$ & $18 \%$ & $18 \%$ \\
\hline & Total & $100 \%$ & $100 \%$ & $100 \%$ & $100 \%$ & $100 \%$ & $100 \%$ & $100 \%$ & $100 \%$ \\
\hline \multirow[t]{3}{*}{ Location } & Intersection & $19 \%$ & $32 \%$ & $40 \%$ & $65 \%$ & $41 \%$ & $46 \%$ & $41 \%$ & $54 \%$ \\
\hline & Link & $81 \%$ & $68 \%$ & $60 \%$ & $35 \%$ & $59 \%$ & $54 \%$ & $59 \%$ & $46 \%$ \\
\hline & Total & $100 \%$ & $100 \%$ & $100 \%$ & $100 \%$ & $100 \%$ & $100 \%$ & $100 \%$ & $100 \%$ \\
\hline \multirow[t]{5}{*}{ Age } & $<15$ years & $4 \%$ & $8 \%$ & $4 \%$ & $12 \%$ & $3 \%$ & $3 \%$ & $0 \%$ & $1 \%$ \\
\hline & 15-17 years & $2 \%$ & $10 \%$ & $3 \%$ & $6 \%$ & $19 \%$ & $32 \%$ & $3 \%$ & $4 \%$ \\
\hline & $18-64$ years & $50 \%$ & $70 \%$ & $51 \%$ & $69 \%$ & $56 \%$ & $62 \%$ & $93 \%$ & $92 \%$ \\
\hline & $65+$ years & $44 \%$ & $12 \%$ & $42 \%$ & $13 \%$ & $22 \%$ & $3 \%$ & $4 \%$ & $3 \%$ \\
\hline & Total & $100 \%$ & $100 \%$ & $100 \%$ & $100 \%$ & $100 \%$ & $100 \%$ & $100 \%$ & $100 \%$ \\
\hline
\end{tabular}

Calculated based on EC 2014 [4] and CARE database [1] 
account when assessing comfort are explored, followed by the explanation of the methodology to address these aspects.

A definition that is relevant especially in view of the implications of ITS solutions for vulnerable road users, comes from Slater [27]. This definition of comfort is: “,,(...) a pleasant state of physiological, psychological, and physical harmony between a human being and the environment" ([27], p. 4). This definition identifies three dimensions - physiological, psychological and physical - relevant for the assessment of comfort according to Slater. Each of these three dimensions must be defined and assessed separately, in order to take the complexity of this issue into account.

A definition of the three dimensions, specifically addressing pedestrian comfort, can be found in [25], where the comfort needs of pedestrians in high density and high complexity urban scenarios are discussed. Sarkar pleads for a two-level approach for the assessment, on a micro and a macro level ([25]: pp. 6): the macro level encompasses the general circumstances and the infrastructural context including relevant standards and criteria, referred to as "service levels". The micro level focusses on the actual quality of the task and on factors that directly influence the individual perceptions referred to as "Quality levels". This broadens the definition by Slater, as the micro level specifically addresses individual circumstances under which a certain mode is used or has to be used and allows for a more specific assessment of the actual quality of certain situations. This indicates that comfort assessment varies by the transport mode used, as there are specific differences concerning the physical, psychological and physiological work load. To assess work load appropriately, infrastructural, societal and individual circumstances need to be taken into account for the respective mode. While there is also a reference to the accommodation of pedestrian needs and the psychological level of comfort, no specific tools to assess this dimension other than walking speeds and the ability to engage in social interactions are specifically discussed.

A study focussing on the qualitative aspect of (pedestrian) comfort, while defining comfort similarly, includes the emotional component more explicitly:

“(...) comfort for pedestrians is a positive emotional reaction to external surroundings (the walking environment) in different situations, including physiological, physical, social and psychological reactions." ([21]: p. 2). The emotional component of comfort is considered as: “(...) short-lived emotional reactions rather than cognitive reflections (...)" ([21], p. 2). This approach implies a stronger focus on individual assessments of comfort that is influenced by both external and internal factors. The actual questions that [21] included in their study focus on "thermal comfort, visual comfort, acoustic comfort, tactile comfort, smells, air pollution and allergens, the ease to move and the feeling of security" ([21], p. 2). They also introduce the concepts of efficiency and perceived safety which opens the discussion not only to infrastructural aspects and indicators such as walking speed, but to characteristics that help to specify certain needs in relation to individual preferences and (in)abilities.

The research project PROMPT, focussing on pedestrian comfort needs, shows the importance of considering individual characteristics such as age, gender and state of health, especially in the connection with mobility impairments, as essential factors for assessing comfort.

The aspects of comfort that the evaluation methodology identified are:

- Physiological, psychological and physical harmony ("harmony");

- Quality and service levels; and

- Internal and external factors.

The relationship between these aspects are:

- Service levels refer to the external factors. This is the external environment, and is thus the link to the physical harmony

- Quality levels refer to the internal factors. This is the physical efforts and the perception of the environment, and the link to the psychological and physiological harmony.

Thus, a method must address the quality and service levels as defined above in order to assess comfort.

A model provided by [15] gives insight into frameworks on how to generally assess mobility, focussing specifically on motorised transport. Units of measure, modes, performance indicators, consumer benefits as well as land use, and improvement strategies are also viable for the assessment of vulnerable road user mobility. Examples include person miles travelled, the number of person trips, and travel convenience.

The methodology has to consider all the relevant aspects of comfort. The comfort level of different vulnerable road user groups should be assessed and the specifics of each vulnerable road user group should be taken into account appropriately. A combination of qualitative and quantitative instruments was envisaged.

A combination of approaches for assessing comfort has been developed by $[18,21]$ for pedestrians.

$[21,22]$ also applied survey methods such as focus group interviews and in-depth interviews to cover these aspects. In addition, they applied quantitative checklist methods to rate available infrastructure (i.e., pavement conditions, continuity of sidewalks/cycle paths, seatings, etc.) on different scales. This combined approach that integrates both survey methods for individual road user ratings and observations for 
infrastructure assessments and road user behaviour, provides insight into the quality and service levels that determine the comfort of different vulnerable road user groups.

When trying to connect general concepts of comfort, such as walkability, cyclability, etc. to ITS solutions and their potential impacts on the travel comfort of vulnerable road users, not only systems directly aimed at improving comfort are relevant but also those that are focussed on improving safety. The studies above show that objective as well as perceived safety play an integral role in the individual comfort perceptions. Systems that allow vulnerable road users to identify potentially critical scenarios in traffic and to avoid them, by being warned, routed or re-routed based on current traffic situations and/or general conditions, are directly related to the comfort concept.

\subsection{Development of a mobility and comfort methodology to take into account vulnerable road users}

The challenges that need to be addressed to develop the mobility and comfort impact assessment methodology are similar to those of the safety impact assessment with respect to the focus on vulnerable road users. These are:

- There are very few methods available to assess mobility and comfort, and of vulnerable road users, specifically.

- There is a little or no data is available on the mobility of vulnerable road users, and even less on the comfort of vulnerable road users (discussed in section 4.3).

The TeleFOT framework [10] for assessing mobility and comfort identified characteristics in transport that influence mobility (amount of travel and travel patterns) and comfort (journey quality). It provides a structure for assessment. The TeleFOT framework is shown in Fig. 3. The aspects of mobility and comfort are embodied in this framework. Usefully, it identifies indicators that can be quantified, as shown in the boxes at the left hand side of Fig. 3. The TeleFOT approach provides the starting points for the assessment of each ITS: function, design, use case, and types of impact.

The nine mechanisms in the eIMPACT methodology are used to guide the assessment in covering all aspects of the potential type of impact. The aspects from the general description of mobility and comfort are investigated by the mechanisms as described below:

For mobility:

- The number, duration and length of journeys is covered by mechanism 6

- Modal choice is covered by mechanism 7

- Route choice is covered by mechanism 8 .

- The timing of trips is covered by mechanism 6
For comfort, workload, stress, uncertainty and safety perception are addressed by mechanisms 1 and 2 .

Mechanisms 3, 4 (relevant for comfort) and 5 (relevant for comfort and mobility) are indirect and can be positive or negative.

Mechanism 9 is not relevant.

\subsection{Data}

\subsubsection{Data on mobility}

Mobility can be assessed based on the following variables defining mobility outside of the house [24]:

- Region (urban, rural, etc.)

- Number of trips per person

- Mode of transport

- Main mode of transport (for a specific trip [purpose])

- Modal-Split (of all modes used on a trip, including intermodal trips)

- Trip purpose

- Trip duration

- Route choice

- Travel distance

- Travel length

- Travel speed

- Time spent on travelling, duration

- Number of journeys

- Departure time/arrival time

While there a number of mobility studies on all levels, regional, national and European, standardised data for comprehensive mobility behaviour assessment are scarce.

One source has been found though within the Cost-action Survey harmonisation with new technologies [26]. The purpose of the Cost-action was to coordinate research efforts on data harmonization for transport surveys across Europe. The action contains mobility data for the following road user groups: Walking, Bike, Moped and motorcycle, Car driver, Car passenger, Car or Moto (in Spain), Public transport and others.

The following countries are included: Belgium, Denmark, Finland, France, Germany, Great Britain, the Netherlands, Norway, Sweden and Switzerland. Travel data divided by purpose such as "Commuting - work and education" and "Other" are available for the general road user groups, but not available for different age groups. The data per general road user group (i.e. not divided in age groups) are for all persons (weighted by population). The travel data per age group is by mode and age group only regarding travellers (=persons that have been mobile on the survey day(s)). 
Fig. 3 Mobility and comfort model, developed from the model found in the TeleFOT project, Impacts on Mobility - Results and Implications [11]

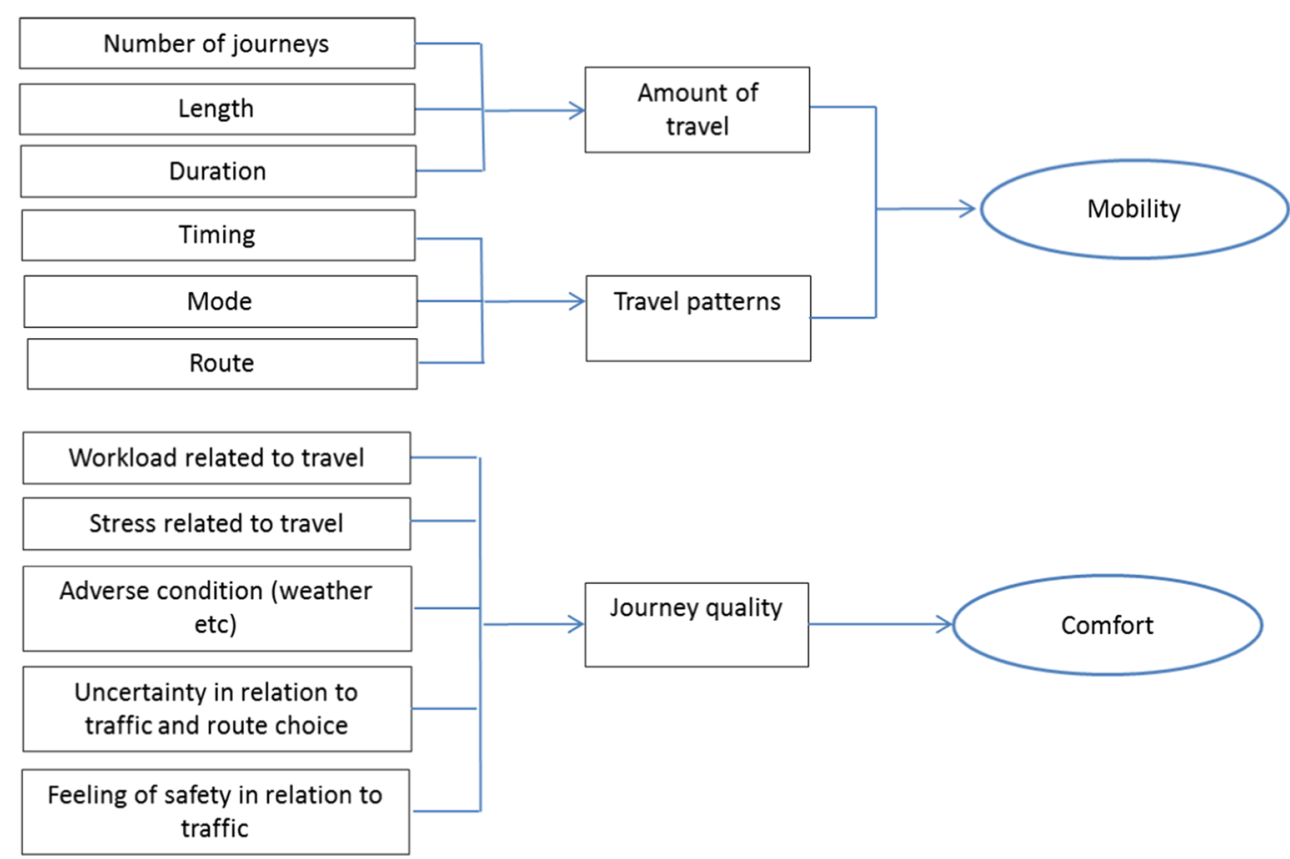

There are examples of other national or local travel surveys with deeper or more detailed travel information; but those surveys are linked to context and are not transferable to transport in Europe as a whole.

\subsubsection{Data on comfort}

Most projects dealing with aspects of walkability, cyclability and the preconditions for Power Two Wheelers usually provide data for general comfort assessment. These assessment approaches vary by project goals, applied data collection methods and vulnerable road user group. Due to the nature of the construct "comfort", data on comfort is strongly based on qualitative data, frequently collected with the help of road user questionnaires and observations in the public space, or based on check list rankings.

The EU project PROMPT [23] provides a usable database based on case studies on pedestrian comfort conducted in 6 EU countries, including France, Switzerland, Norway, Belgium, Finland and Italy. Comfort is assessed on the micro- and macro levels discussed in Section 4.1, and the data were collected via interviews with pedestrians. The PROMPT study is also the only available study that provides data on pedestrian comfort that allows cross-country comparisons. The data collected provides a good overview of urban areas, including residential and suburban areas. The data were collected based on a specially-developed pedestrian comfort questionnaire, taking both psychological as well as external, physical factors into account.

The results presented by [22] cover a wide range of indicators, including attractiveness and social aspects, and serve as the basis for a comprehensive evaluation of the comfort assessments of the respondents in the participating countries. However, these kind of data are only available for the participating six partner countries and is specifically focussing on pedestrians, not taking cyclists and Power Two Wheelers into account. A solid data basis for assessing comfort needs to provide information on the other vulnerable road user groups as well, and it should allow cross-country comparisons at the European level.

The comfort effects that were assessed in the VRUITS are:

- workload related to travel;

- stress related to travel;

- uncertainty related to travel;

- travelling in adverse conditions (weather etc.);

- feeling of safety in relation to traffic.

The assessment of exposure of pedestrians and bicyclists in different types of locations and traffic environments was based on a Swedish study by Gustafsson and Thulin [7] and on the travel survey data from [26]. The Swedish study was based on exposure data from the Swedish transport safety survey TSU92- for the years 1998-2000. TSU92- was questionnaire-based and a continuously running national survey focused on the traffic environment exposure of vulnerable road-users, where they gave information regarding what type of locations and traffic environment they travelled in, together with distances travelled and time spent on travelling. The Swedish study was the only one found regarding exposure of pedestrians and bicyclists in different types of locations and traffic environments. The Swedish data were therefore 
used as a starting point in the assessment of the total EU-28. The estimations of exposure were used to draw the numerical estimates of the effect on comfort by the ITS in the total transport system.

\subsection{Summary}

The method to assess the mobility and comfort impacts of ITS on vulnerable road users was developed from the method introduced by Kulmala (2010), initially developed for the assessment of safety impacts of ITS for cars. The method for mobility and comfort impact assessment followed the steps defined by Kulmala (2010), but when assessing mobility and comfort numbers 1-5 (comfort) and 6-8 (mobility) were of interest. The method was enhanced and adjusted to take into consideration the mobility and comfort of vulnerable road users covering: mobility and exposure of vulnerable road users measured in: trip length, duration and frequency; and comfort of vulnerable road users measured in: how the users perceive their travel, i.e., change their opinion regarding the comfort of the travel undertaken, and perceived safety in relation to traffic.

\section{Application of the methodologies}

The application of the safety, mobility and comfort methodologies followed the same set of steps through the qualitative and quantitative assessments. Figure 4 illustrates the steps taken. The methodology presented in this paper corresponds to the light green box labelled "Quantitative assessment 10 ITS" in Fig. 4. For completeness, this section describes all the steps in the process. The reduction from 23 systems in the qualitative assessment to 10 systems in the quantitative assessment was necessary to keep the work feasible within the time and budget of the project. Milestone Report 2 [13] provides detail on the process used to select the 10 systems for quantitative assessment. Below, the application of the steps is described in more detail.

\section{System descriptions}

Comprehensive and clear system descriptions were required for the assessment, especially for systems that did not yet exist on the market. This includes the functional and technical description and limitations of systems, anticipated user reactions ${ }^{1}$ and the expected effects of systems on safety, mobility and

\footnotetext{
${ }^{1}$ This is due to the fact that assessment is prescriptive, that is, the systems do not exist yet or for which no empirical data is available.
}

comfort of road users. Examples of issues covered in the system descriptions are the following:

- Description of the purpose and technical performance of the system

- Description of the safety, mobility and/or comfort issue addressed by the system

- Description of the type of safety, mobility and comfort aspects the system affects. For safety, these are accidents the system aims to prevent or a description of type of accidents consequences the system aims to mitigate

- Description of circumstances in which the system works or is assumed to work or does not work

- Expectation of effects on the behaviour of the driver or other road users; effects on safety, mobility and/ or comfort, such as anticipated driver reactions and vulnerable road user reactions.

2. Description of safety, mobility and comfort effects

The relevant safety, mobility and comfort mechanisms were identified and applied to each investigated ITS. This includes description of expected changes in driver and vulnerable road user behaviour and documentation of the expected effects based on existing literature and other evidence available. This other evidence included already available empirical evidence on safety, mobility and/or comfort impacts of systems with partly similar functionalities and indirect evidence on safety, mobility and/or comfort impacts such as more general assessment of the effects based on knowledge of driver/vulnerable road user behaviour, traffic flow, and effects of comparable systems.

3. Selection of systems for more detailed assessment

Based on the qualitative assessment, the systems were prioritised. The selection of systems for the final quantitative assessment took place in a VRUITS workshop in June 2014, with experts in the field of traffic safety, ITS, traffic planning and vulnerable road users [13]. Multicriteria analysis was used to identify the systems to be included in the quantitative analysis. The result from the workshop is a reduced list of ITS for further detailed quantitative analysis.

4. Estimation of effects by mechanism

In this step the earlier effects of each safety, mobility and comfort mechanism from step 2 were refined in terms of percentage increase/decrease of relevant accidents or exposure of vulnerable road users such as, trip length, duration and frequency, and comfort of vulnerable road users such as perception of travel; i.e. change in opinion regarding the comfort of the travel undertaken, and perceived safety in relation to traffic. The reference case for the estimates was the situation without any ITS. 
Fig. 4 Steps in applying the methodology

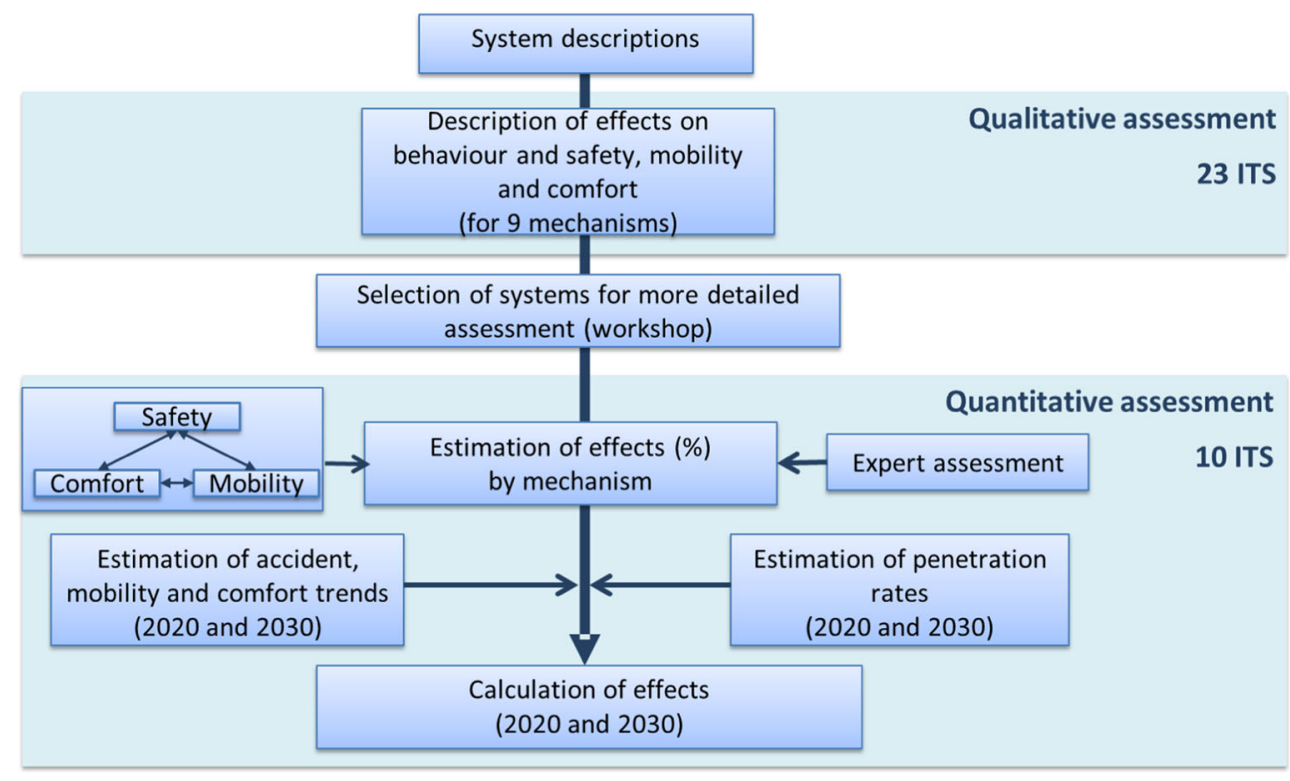

The first qualitative estimates of the effects were generated by the responsible partners (one per system) who studied the relevant literature and system functioning in detail. Lack of empirical results, qualitative or quantitative, was a significant issue because many of the selected ITS are still in development, and hence little was known. Furthermore, findings may depend significantly on the cultural and legal context, and on other circumstances (like level of urbanization, climate, etc.). It was most often not possible to find literature on direct or indirect effects, and, when it was available, it was only for specific countries or circumstances.

Mechanism 9 was not relevant for any of the ITS under consideration.

The qualitative estimates made by the responsible partners were reviewed by the safety experts and mobility and comfort experts among the consortium partners to crosscheck and validate the estimates. This crosscheck and validation of estimates was found especially important for assumptions for which no literature existed.

External experts were used to modify the safety, mobility and comfort estimates. One thousand experts in Europe, the US and Japan were contacted to fill in a webbased questionnaire during the period June 26-August 10, 2014, in addition to the experts within the VRUITS project.

Altogether, 77 answers to the questionnaires from experts outside the project were collected from at least 19 different individuals representing experts in the areas of road safety, vulnerable road users, engineers, ITS developers, human factors, urban mobility and risk analysis from Austria, Finland, Germany, Italy, the Netherlands, the UK, Sweden, Spain, Switzerland, cycling associations around the world as well as the European Motorcyclists association.
The qualitative assessments and external expert assessments (1-13 per system) were used as background information for the next step, when responsible partners estimated the quantitative effects for each relevant mechanism for each system. The input of the external experts was used to check whether the assumptions made in the earlier phases of the assessment were correct and as background information when generating the numerical estimates. The quantitative estimates for safety, mobility and comfort were reviewed by all partners to crosscheck and validate the quantitative estimates.

In addition, the interaction between safety, mobility and comfort was taken into account. For example, an increase in mobility increases exposure, which, for safety, affects the safety assessment. Ultimately, a numerical value for the effect on safety, mobility and comfort was established for each mechanism, for each relevant road user group.

5. Calculation of effects for $100 \%$ equipment rate

The effect estimates for safety, mobility and comfort were used to calculate the overall low, medium and high estimates of the effect of the system. The calculations included five steps, namely:

- Identification of the main classifying variable: A main classifying variable is an aspect that is the most important in the effectiveness of the system. Systems can perform better under some circumstances than others, for example, a system that works only at an intersection does not have an impact at road sections that are not intersections. Because systems sometimes work in several circumstances, the most important circumstance is chosen, and made the "main classifying variable". The main classifying variable is used as a weight when quantifying the effects under different 
circumstances. For example, suppose that the ITS under assessment was more effective on preventing pedestrian accidents than cyclists accidents. If the system was estimated to prevent $30 \%$ pedestrian accidents and $9 \%$ of multiple vehicle accidents involving cycles, then the overall effect was determined by multiplying the share of relevant accidents by these effect estimates, and summing the results. In the example, this would give for the overall estimate the value " $30 \% *$ share of pedestrian accidents $+9 \% *$ share of multiple vehicle accidents involving cycles". These outcomes are used in step 6, where the effectiveness per situational variable is combined with the safety, mobility or comfort data, which is split into situational variables.

- Determine the estimates per mechanism as described in step 4

- Combine the estimates per mechanism into an overall estimate: First, the estimates given in percentages were converted to coefficients of efficiency (e.g. a decrease of accidents by $10 \%$ means that the target group of accident is multiplied by coefficient 0.90 ). Secondly, the total effect was computed by multiplying the coefficients for each mechanism and giving this total effect as a percentage.

- Apply reduction factors for usage and penetration rate: The estimated non-usage of systems (e.g. due to annoyance) was taken into consideration together with the penetration rate, as factors reducing the effect at $100 \%$ equipment rate.

6. Combine the overall effect with accident, mobility and comfort data

The overall estimates per system were applied to the relevant data (road accident data, mobility and comfort data) for the EU-28. The calculations were carried out by using an Excel tool which structured the relevant data and effect estimates.

7. Calculation of effects for estimated penetration rates in 2020 and 2030

The target year estimates were calculated by using the estimated penetration rates of the selected ITS systems for the years 2020 and 2030, documented in [6], and taking into account the calculated accident, mobility and comfort trends. The years 2020 and 2030 were chosen because many European goals and calculations are for these years. The impacts were assumed to be linear with the respective penetration rates, unless otherwise indicated. The linearity is logical when only the infrastructure or the vehicle or the vulnerable road user be required to be equipped, all other factors being kept constant.

Even though the data allows the presentation of the results quantitatively, a certain degree of uncertainty exists related to the results. In general, we can have uncertainty related to a) estimates of safety, mobility and comfort effects (depends on the results of expert assessment and findings from literature), b) accident, mobility and comfort data (e.g. for some systems we might have better data related to accident types the system aims to prevent than to some other ones), and c) estimated accident, mobility and comfort trends and penetration rates. The range of uncertainty related to each of these varies according to the system under investigation and thus it is not possible to provide any estimate on the general uncertainty of our assessments before the exact systems to be assessed are known.

The uncertainty in the safety effects were addressed by providing low, average and high values for all the estimates and each relevant safety mechanism. Similarly, the estimates of penetration rates included low, medium and high values. Uncertainties in accident data and accident forecasts were not addressed. During the assessment process it became clear that the yearly number of injuries reported to the CARE database and to national databases does not correctly reflect the situation in reality. The underreporting of injuries is common and the extent of this problem varies among countries. [] contains more details. Therefore, the results regarding injuries should be interpreted with caution and considered as an indication of the effect. For fatalities the data are of better quality but not perfect either.

\section{Reflections on the application of the methodology}

\subsection{Safety assessment methodology successfully modified that of Kulmala (2010) to take into account vulnerable road users}

The safety assessment conducted in the VRUITS project was the first time that the safety impact assessment framework of [15] was applied to calculate the safety effects for vulnerable road users. This project primarily assessed the effects of new systems which have not been yet used in real traffic (systems were not in production or on the market). Effort was invested in creation of detailed descriptions of the functionalities of each system and several experts were involved in finalising the description and specifying the functioning of each system. Next, literature was reviewed to develop valid and reliable estimates of the effects. Usually it was difficult to find any direct evidence about the effectiveness of the systems and therefore we needed to combine several types of evidence from different sources to understand the user context and road user's potential reactions to systems. To increase the reliability 
of the estimates, several experts contributed to the work in parallel and the estimates were cross-checked.

Numerical estimates were produced by using the European Risk Calculation tool ERiC whose structure and content was specifically modified for the assessment of safety effects for vulnerable road users. The main modifications concerned the collection and modification of European-wide accident data in order to calculate the effects for the EU-28. The assessment exploited the statistics from the CARE database, but the content was not detailed enough for our purposes without additional modifications. The variability in the quality of the accident data entered into CARE by country was tackled by grouping the countries into three clusters which were formed based on the prevalent safety situation in each country (countries with a similar safety situation were included in the same cluster).

The method is a systematic approach to cover all effects of the systems; including not only the expected positive effects of the systems but also the indirect and negative effects. Compared to previous applications of the safety assessment method of ITS [15], mechanism 9 (modification of accident consequences) was not relevant for the systems for vulnerable road users in our analysis. Mechanism 9 only applies to systems that affect the outcome of an accident that has already occurred, such as eCALL. None of the systems investigated here had this attribute.

\subsection{A new method to assess mobility and comfort was developed and applied}

A new method was developed in this project to assess the mobility and comfort impacts of ITS systems on vulnerable road users. The method is analogous to the safety approach. The problematic aspect of applying the method was data availability, especially for comfort. The use of expertise, external as well as internal to the project to assess the impact, was a useful method to steer the assessment process. The methodology provides a good starting point for future work and methodological development.

The comfort and mobility impact assessment process was conducted for different road user groups, and for different age groups. Comfort has not been covered in previous studies to any great extent, especially not with the focus on vulnerable road users in relation to ITS. Therefore the assessment regarding comfort could not be based on evidence from literature or previous studies; the assessments had to be based on similar studies, if available, based on car drivers' comfort in relation to ITS, or assessments from the experts within the consortium or the responses to the expert questionnaire. The concept of mobility is much more common in literature, but still the existing studies do not often cover mobility of vulnerable road users, and seldom in relation to new ITS.
Despite the limited availability of literature regarding mobility and comfort of vulnerable road users in relation to new ITS, much literature was reviewed to have as valid and reliable estimates as possible of the estimates of the effects. To increase the reliability of the estimates, several experts within the project contributed to the work in parallel and the estimates were cross-checked.

The eIMPACT methodology provided a systematic approach to cover all mobility and comfort effects of the systems; including not only the expected positive effects of the systems but also the indirect and negative effects.

The comfort benefits, the assessment of which was conducted in an experimental manner in this study, form a considerable part of the total vulnerable road user benefits for some systems, although safety benefits usually outweigh all other positive impacts [1]. This leads to the need for a standardised methodology and framework for vulnerable road user benefits assessment and especially for the assessment of comfort benefits. However, the proper application of an overall standardised methodology for benefits assessment requires the collection of harmonised safety and mobility data across EU countries, to be set at a level of detail sufficient to disaggregate system benefits not only to general vulnerable road user groups but also per age category.

Although not addressed in this study, the health benefits of active modes (walking and cycling) can have an impact on comfort levels and they are seen to outrun accident risks. ITS applications could be useful in improving the perception of these health benefits. Future analyses could take health benefits into account.

\subsection{Validation of the methods}

Because the systems analysed in VRUITS are not yet deployed, the possibility to validate the methods lies in the future. The safety impact assessment utilized methods described in detail in the literature. The mobility and comfort methodology was developed in this project. The VRUITS deliverables D3.1 [1] and D3.2 [16] make references to the relevant literature on approaches and formulae used. Chapter 2.3 of D3.1 [1] describes the procedure applied and clarifies calculations with examples. All assumptions and results used as input are made transparent for each ITS analysed (Chapters 4-12 of [1]) which makes it possible to compare the findings with earlier (and future) assessments. The transparency will enable the validation of the results in the future. The validation could be done in the future field operational tests focusing on measuring user behaviour, as it was done in DRIVE C2X project $[11,31]$. In addition, new findings can be incorporated into the assessments. However, the detailed calculations were not included in this paper. 


\subsection{Lessons learned}

In order to improve the accuracy of the estimates, there is a need for better accident data (on number and details of accidents, including hospital records; especially regarding injuries), also to correct for injury underreporting. Also mobility data (such as quantitative detailed information about trip length, duration and frequency for vulnerable road users of different groups and age groups), and comfort data (such as how the users perceive their travel; i.e. change their opinion regarding the comfort of the travel undertaken, and perceived safety in relation to traffic) is needed. Finally, trials to test the functioning of the systems and their effect on road user behaviour are needed to better understand the effects of the systems.

Acknowledgements The authors are in debt with the members of the research team of the VRUITS Project. The VRUITS project has received funding from the European Commission Seventh Framework Programme (FP7-TRANSPORT-2012-MOVE-1) under Grant agreement No. 321586.

Open Access This article is distributed under the terms of the Creative Commons Attribution 4.0 International License (http:// creativecommons.org/licenses/by/4.0/), which permits unrestricted use, distribution, and reproduction in any medium, provided you give appropriate credit to the original author(s) and the source, provide a link to the Creative Commons license, and indicate if changes were made.

\section{References}

1. Bax B, Giannelos I, Mans D, Silla A, Leden L, Rämä P, Scholliers J, Johansson C, Van Noort M, De Kruijff J, Malone K, Woldeab Z, Hancox G, Bell D, Garcia Melendez A, Martin O (2016) Assessment of selected ITS applications on safety, comfort, mobility and socio-economic impact. VRUITS project deliverable D3.1

2. CARE (2009) EU roads accident database

3. Draskóczy M, Carsten OMJ, Kulmala R (1998) Road safety guidelines. CODE Deliverable B5.2

4. European Commission (EC) (2014) EU transport in figures. Statistical pocketbook 2014. http://ec.europa.eu/transport/factsfundings/statistics/doc/2014/pocketbook2014.pdf. Accessed 8 Oct 2014

5. European Transport Safety Council (2003) Transport Safety Performance in the EU: A Statistical Overview, http://etsc.eu/wpcontent/uploads/2003 transport safety_stats_eu_overview.pdf

6. García Meléndez A, Pérez Martín O, Morris A, Hancox G, Bell D, van Noort M (2014) Implementation scenarios. VRUITS project deliverable D2.3

7. Gustafsson S, Thulin H (2003) Gående och cyklister, exponering och skaderisker i olika trafikantmiljöer för olika åldersgrupper. VTI meddelande 928:2003

8. Hansen G, Morris A, Scholliers J, Silla A, Van Noort M, Bell D (2015) Current and future trends in vulnerable road-user accidents in Europe - why we need ITS solutions. ITS World Congress, Bordeaux, Paper ITS-2833, October 2015

9. Høye A (2011) The effects of Electronic Stability control (ESC) on crashes - an update. Accid Anal Prev 43(3):1148-1159
10. Innamaa S, Axelson-Fisk M, Borgarello L, Brignolo R, Guidotti L, Martin Perez O, Morris A, Paglé K, Rämä A, Wallgren P, Will D (2013) Impacts on mobility - results and implications. TeleFOT deliverable D4.4.3, European Commission, information society and media, $7^{\text {th }}$ framework Programme

11. Innamaa S, Rämä P, Hogema J, Kroon L, Heinig I, Schindhelm R, Ojeda L, Servel A, Visintainer F, Giosan I (2014) Safety impacts of cooperative systems. Internal Deliverable of DriveC2X project

12. Johansson C, Bell D, Garcia Mendelez A, Perez OM (2014) Internal report on assessment of mobility and comfort. VRUITS Internal report

13. Kruijff J, Malone K, Scholliers J (2014) Milestone report 2: minutes of the second interest group workshop. VRUITS

14. Kulmala R (2010) Ex-ante assessment of the safety effects of intelligent transport systems. Accid Anal Prev 42:1359-1369

15. Litman T (2011) Measuring transportation - traffic, Mobility and Accessibility. Victoria Transport Policy Institute

16. Malone K, Van Noort M, Johansson C, Silla A, Rämä P, Leden L, Scholliers J, Bax B, Giannelos I, Hancox G (2016) Impact assessment update. VRUITS project deliverable D3.2

17. Marizwan bin Abdul Manan M (2014) Factors associated with motorcyclists' safety at access points along primary roads in Malaysia. Lund, Lund University, Faculty of Engineering, Doctoral Dissertation

18. Methorst R (2003) Assessing pedestrians' needs. The European COST 358 PQN project. http://www.walk21.com/paper search/results_detail.asp?Paper=380. Accessed 16 Jan 2013

19. Nilsson G (2014) Traffic safety dimensions and the Power model to describe the effect of speed and safety. Bulletin 221, Department of Technology and Society, Lund University, Sweden

20. Ö̈̈rni R, Luoma J (2016) Realised Safety Impacts of Electronic Stability Control in Finland IET Intelligent Transport Systems Submitted in 2016

21. Ovstedal L, Ryeng EO (2002) Understanding pedestrian comfort in European cities: how to improve walking conditions? European transport conference Proceddings 2002. Association for European Transport

22. Ovstedal L, Ryeng EO (2004) PROMPT. WP3: Pedestrian Comfort Synthesis Report SINTEF Technology and Society Roads and Transport, November 2004

23. PROMPT (2004) http://prompt.vtt.fi/, http://virtual.vtt. fi/virtual/prompt/comfort.htm

24. Sammer G, Klementschitz R, Kohla B, Meschik M, Herry M, Sedlacek N, Tomschy R, Rehrl K, Schneider C, Fellendorf M, Reiter T, Karmasin H, Wolf E (2011) Handbuch für Mobilitätserhebungen KOMOD. Konzeptstudie Mobilitätsdaten Österreichs V1 Bundesministerium für Verkehr, Innovation und Technologie Abteilung Mobilitäts- und Verkehrstechnologien November 2011

25. Sarkar S (2002) Qualitative evaluation of comfort needs in urban walkways in major activity centers. TRB Annual Meeting, TRB, Washington

26. SHANTI (2014) Post-harmonised travel data collected in the EU Cost Action:TU0804

27. Slater K (1985) Human comfort. Charles C. Thomas Publisher, Springfield

28. Van Noort M, Malone $K$, Silla A, Leden L, Rämä $\mathrm{P}$, Innamaa S, Johansson C, Bell D, Giannelos I, Mans D, van Schijndel M, Morris A (2014) Assessment methodology. VRUITS project deliverable D2.2

29. Winkelbauer M, Riegler S, Völker T, Steininger C, Spyropoulou I, Yannis G, Golias J (2012) Powered two Wheelers - safety measures. Guidelines, recommendations and research priorities. Deliverable D28 of work package 6 . www.2besafe.eu

30. Wimmershoff M, Will D, Pütz A, Lach A, Schirokoff A, Pilli-Sihvola E, Le L, Zlocki A, Sihvola N, Weingart J, Stimming C, Kulmala R (2011) INTERSAFE2, cooperative 
intersection safety. Test And Evaluation Results, Deliverable D8.2

31. Malone K, Rech J, Hogema J, Innamaa S, Hausberger S, Dippold M, Van Noort M, De Feijter E, Rämä P, Aittoniemi E, Benz T, Burchert A, Enigk H, Giosan I, Gotschol C, Gustafsson D, Heinig
I, Katsaros K, Neef D, Ojeda L, Schindhelm R, Sütterlin C, Visintainer F (2014) Impact assessment and user perception of cooperative systems, DRIVE Deliverable D11.4, Grant agreement no.: 270410. 